Supporting Information

\title{
Dual Bond Enhanced Multidimensional Constructed Composite Silicon Anode for High-Performance Lithium Ion Batteries
}

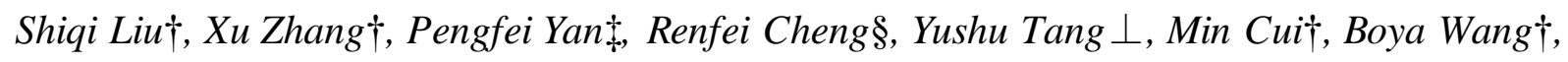
Liqiang Zhang $\perp$, Xiaohui Wang§, Yuyuan Jiang $\ddagger$ Lin Wang $\dagger$ and Haijun Yu*†

$\dagger$ College of Materials Science and Engineering, Key Laboratory of Advanced Functional Materials, Education Ministry of China, Beijing University of Technology, Beijing 100124, P.R. China

Institute of Microstructure and Properties of Advanced Materials, Beijing University of Technology, No. 100, Pingleyuan, Chaoyang District, Beijing 100124, P.R. China

$\S$ Shenyang National Laboratory for Materials Science, Institute of Metal Research, Chinese Academy of Sciences, Shenyang 110016, P.R. China

$\perp$ State Key Laboratory of Heavy Oil Processing, Department of Materials Science and Engineering, China University of Petroleum, Beijing Changping 102249, P.R. China. 
(a)

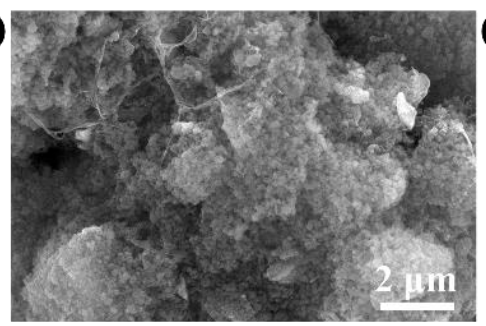

(b)
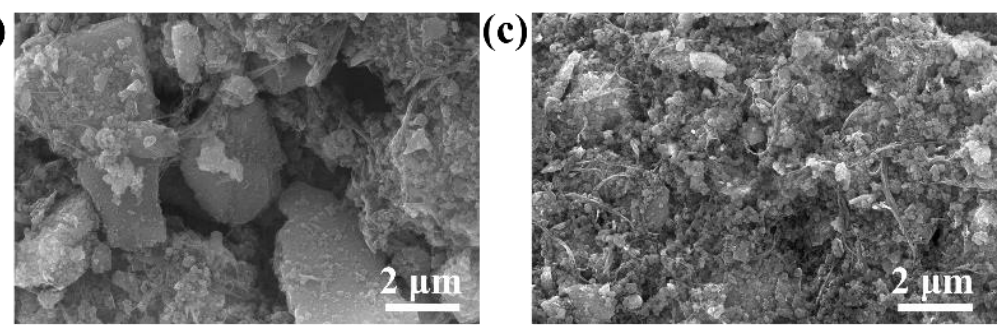

(d)

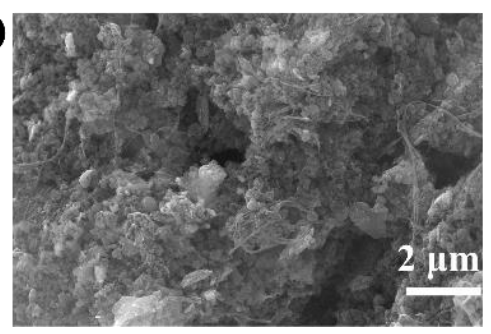

(e)

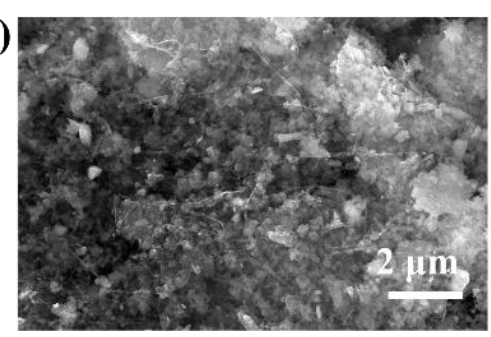

Figure S1. SEM images of (a)-(c) are the ball-milled MSC-80, MSC-40, MSC-20 in differe nt proportions of MXene to nano-Si, respectively; (d) and (e) are the ball-milled MSC using nanoSi by different evolution speed (600 rpm and $900 \mathrm{rpm}$ ), respectively. 


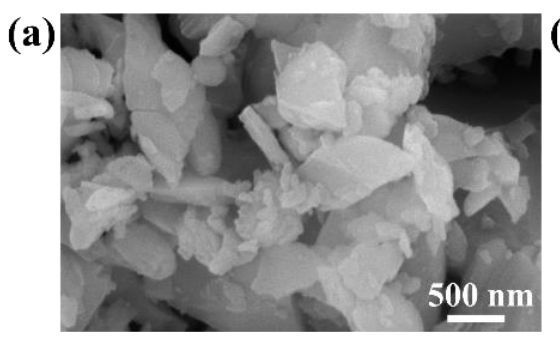

(b)

(d)

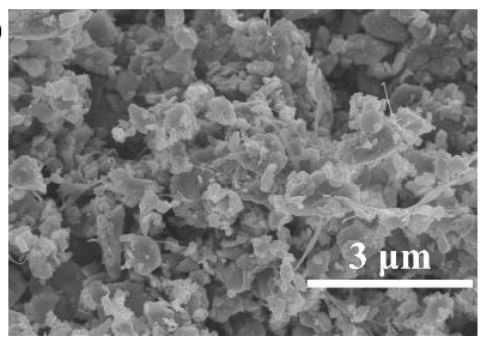

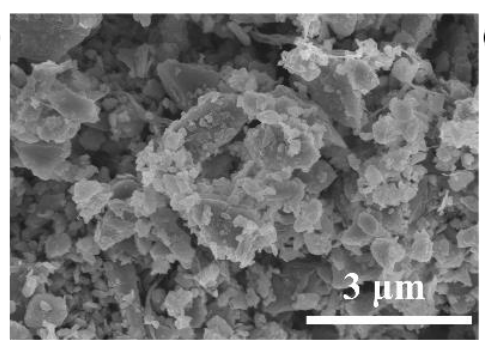

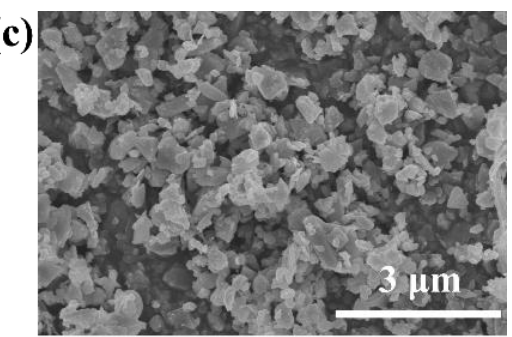

(e)

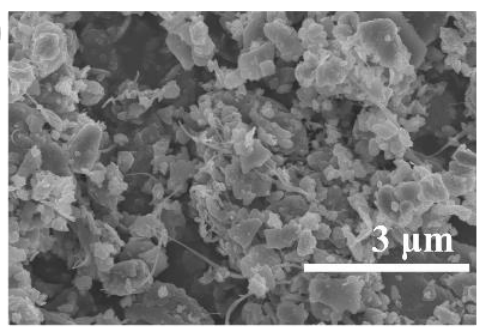

Figure S2. SEM images of (a) micro-Si, (b)-(e) are the ball-milled MSC-80, MSC-60, MSC40, MSC-20 in different proportions of MXene to micro-Si, respectively. 

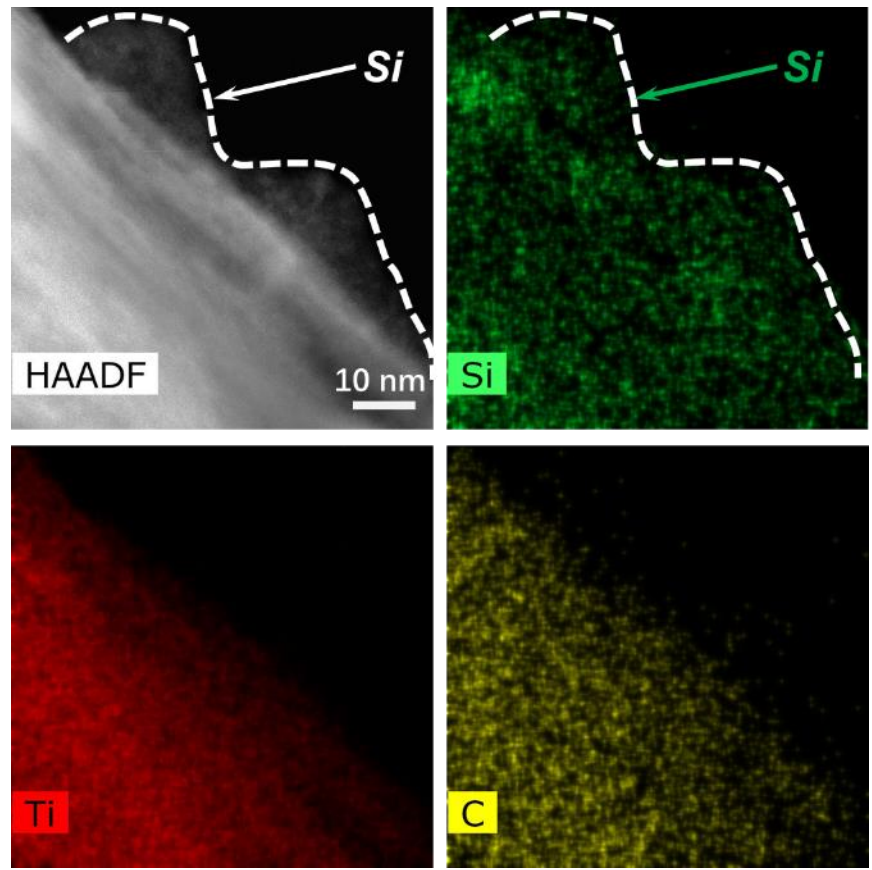

Figure S3. STEM images of the MSC-60 composite consists of MXene and Si with EDX elemental mapping images of $\mathrm{Si}, \mathrm{Ti}$ and $\mathrm{C}$ elements, respectively. 


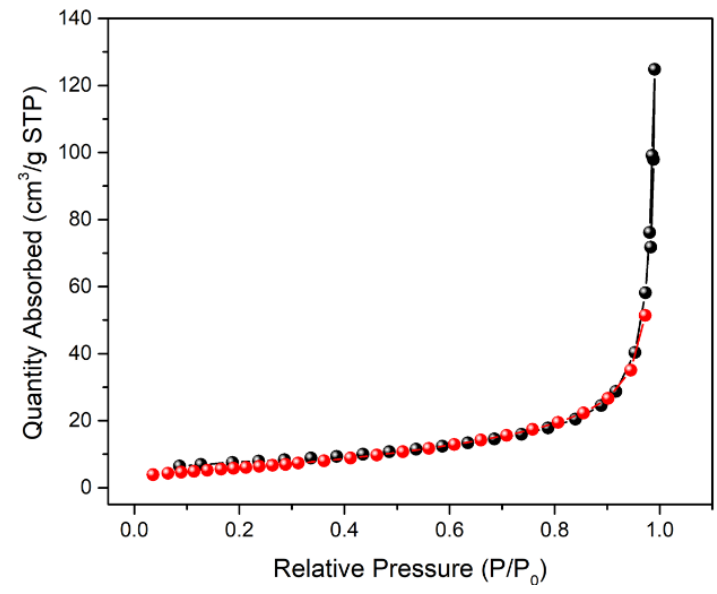

Figure S4. $\mathrm{N}_{2}$ adsorption/desorption isotherm curves of pristine MSC-60. 

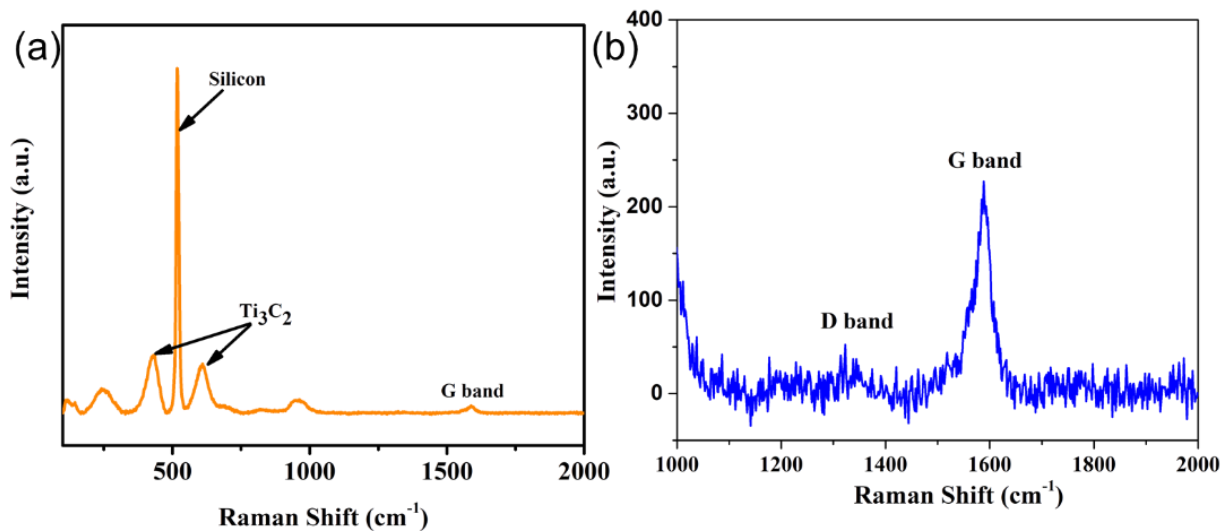

Figure S5. (a) Raman spectra and (b) high-resolution spectra of MSC-60. 
Table S1. XPS peak-fitting results for MSC-60 and MS-60 (showed in brackets)

\begin{tabular}{cccc}
\hline Element & Chemical bond name & Binding energy (eV) & FWHM (eV) \\
\hline Si $2 p$ & Si-Ti & $99.1(99.1)$ & $0.7(0.7)$ \\
& Si-Si & $99.7(99.7)$ & $1.12(1.05)$ \\
& Si-C & $101.0(101.0)$ & $2(1.98)$ \\
& Si-O & $102.8(102.8)$ & $1.86(2.02)$ \\
Ti $2 p 3 / 2$ & Ti-Si & $453.9(453.8)$ & $1.0(1.0)$ \\
& Ti-C & $454.6(454.3)$ & $1.4(1.2)$ \\
& Ti-Ti & $455.8(455.6)$ & $1.8(1.7)$ \\
C $1 s$ & C-Si & $283.4(283.6)$ & $1.17(1.3)$ \\
& C-C & $284.15(284.3)$ & $1.38(1.29)$ \\
\hline
\end{tabular}




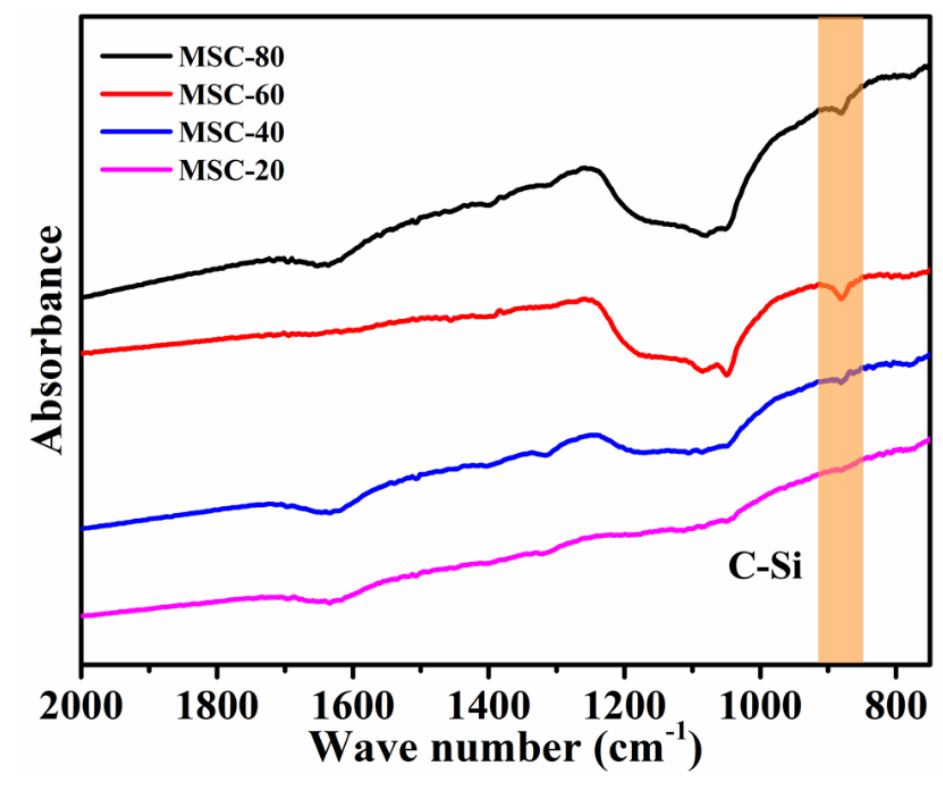

Figure S6. FTIR spectra of the as-prepared MSC composites with different proportions of MXene to Si. 


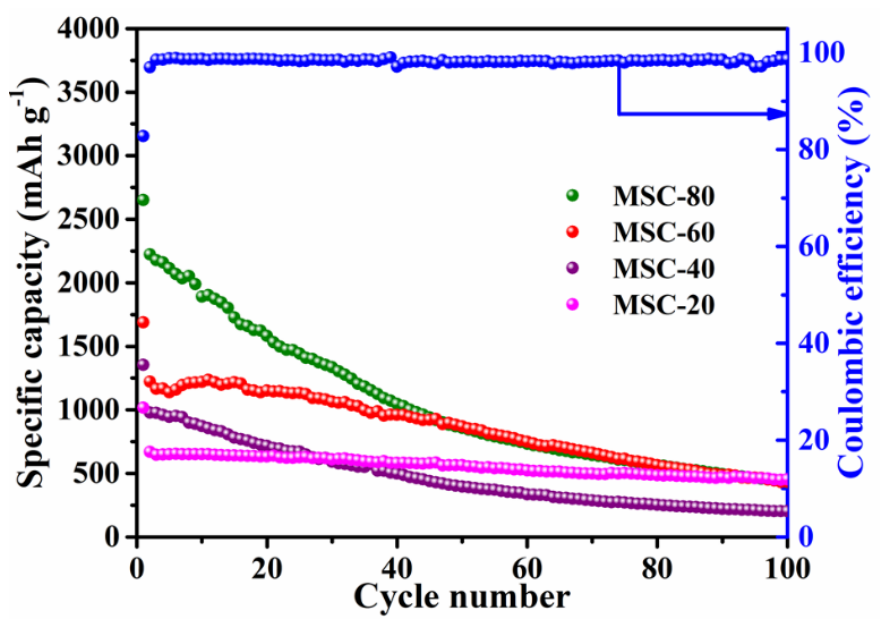

Figure S7. Cycle performances of MSC electrodes with Si microparticles. 

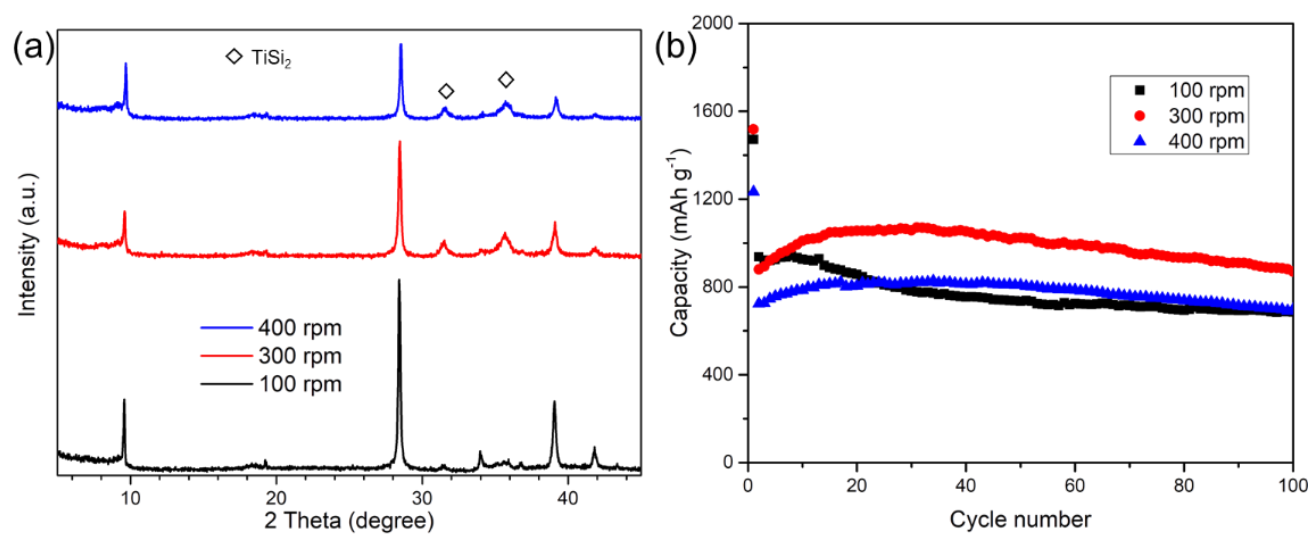

Figure S8. (a) XRD patterns of ball-milled MSC-60 composites prepared at different revolution speeds. (b) Cycling capabilities of the three MSC-60 composite anodes.

The initial reversible capacities of three samples are 961, 953, and $770 \mathrm{mAh} \mathrm{g}^{-1}$, respectively, which show inferior performance than the 200-rpm sample. The poor performance of the 100 rpm sample can be ascribed to the low energy generated from the ball milling process, which is not sufficient for the formation of C-Si and Ti-Si bonds. In other words, the ball milling at 100 rpm is more likely a mixing procedure. It leads to the non-uniform formation of composite materials. In contrast, the revolution speed such as 300 and $400 \mathrm{rpm}$ generate too high energy so that $\mathrm{TiSi}_{2}$ formed inevitably (Figure S8a). As a result, the 200-rpm MSC material shows the superior performance among all materials. 


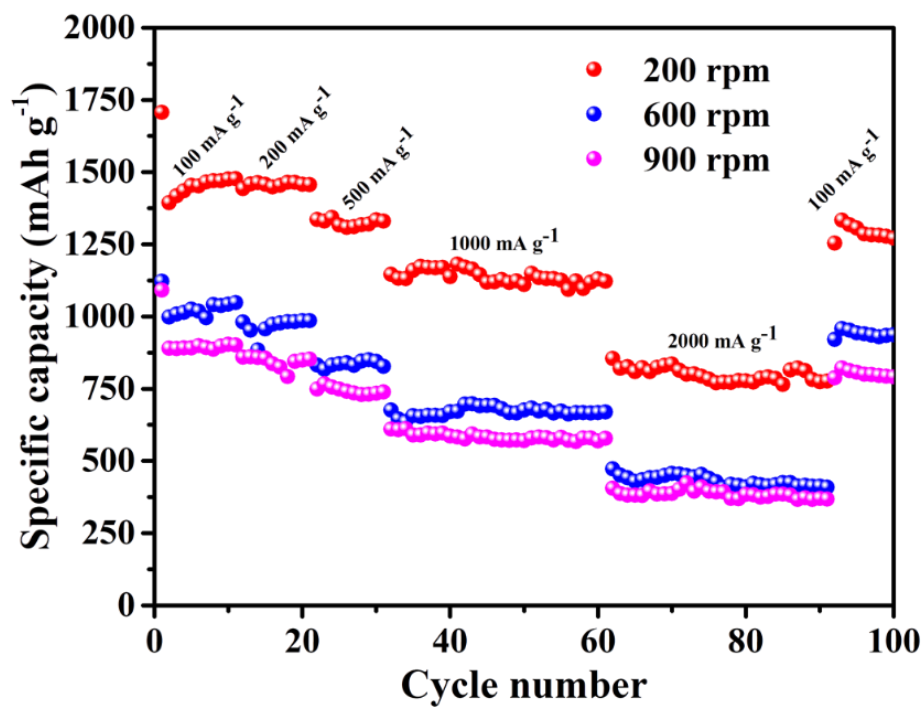

Figure S9. Rate performances of MSC electrodes at different revolution speeds. 
Table S2. Fitting data of EIS curves.

\begin{tabular}{cccc}
\hline & MXene-Si-CNT & MXene-Si & CNT-Si \\
\hline $\mathrm{R}_{\mathrm{s}} / \Omega$ & 2.008 & 2.823 & 3.434 \\
$\mathrm{R}_{\mathrm{ct}} / \Omega$ & 46.28 & 74.05 & 96.25 \\
\hline
\end{tabular}




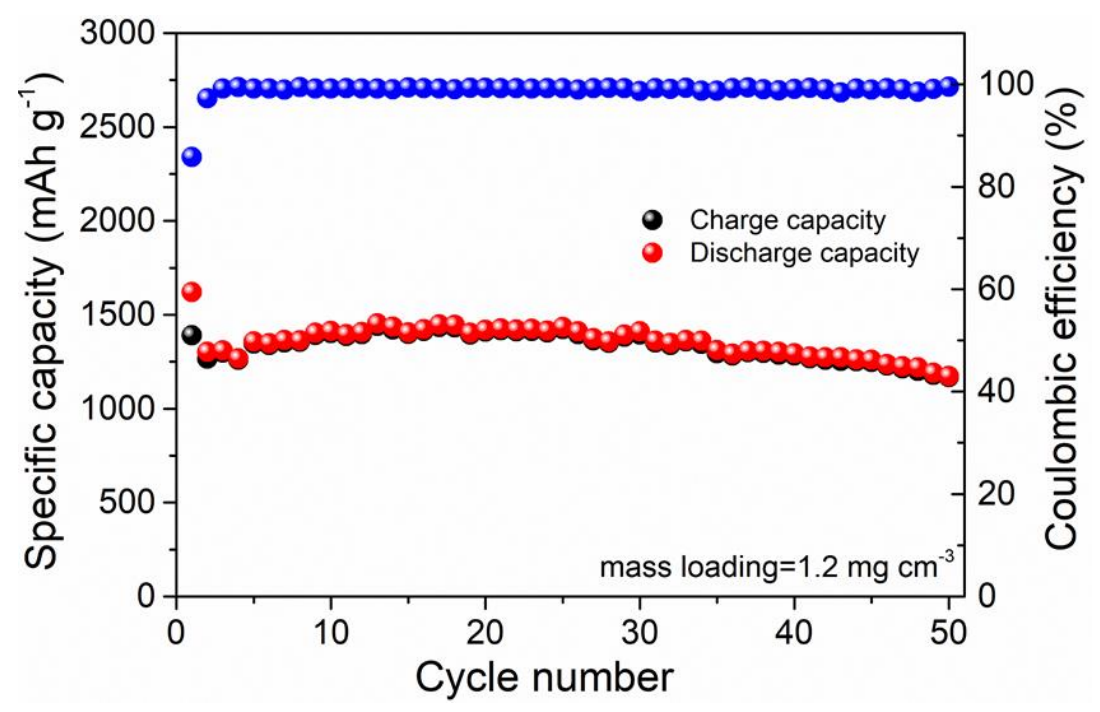

Figure S10. Cycling performance of MSC-60 with electrode composition of 8:1:1 for MSC: Super P: CMC binder. 


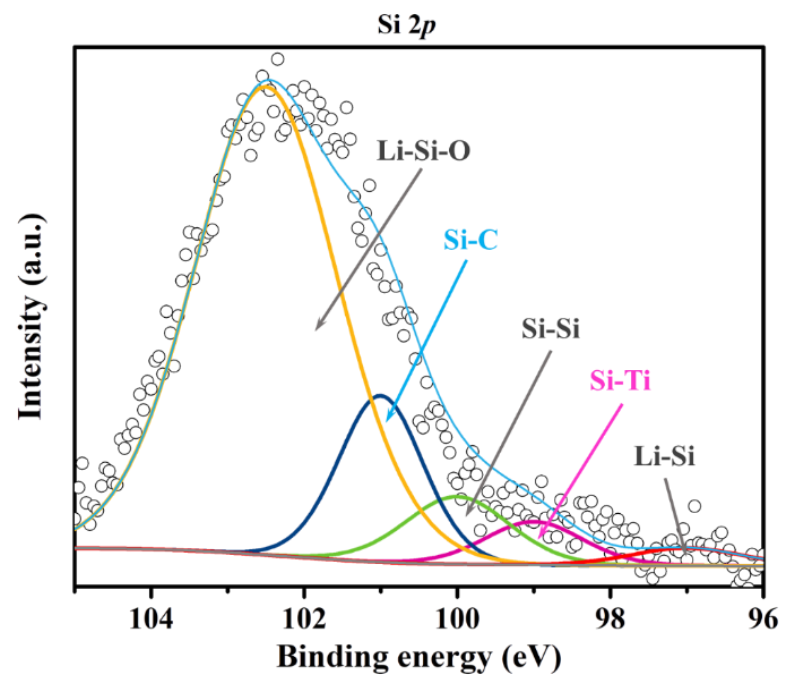

Figure S11. High-resolution XPS Si $2 p$ spectra (after sputtering with $\mathrm{Ar}^{+}$ion) of MSC electrode after 150 cycles. 


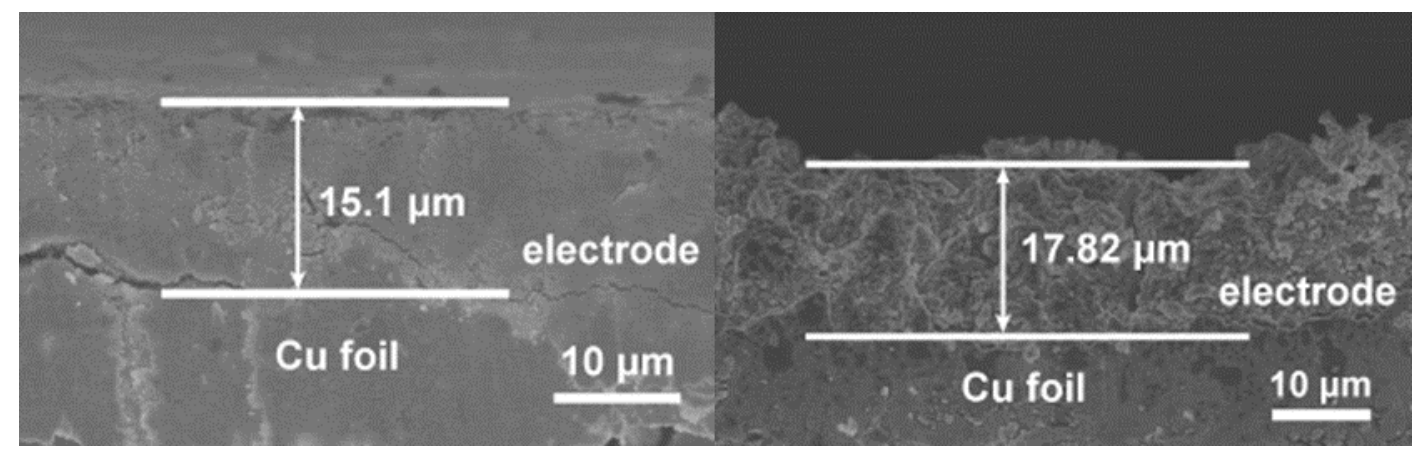

Figure S12. The thicknesses of (a) pristine electrode, and (b) the electrode after 300 cycles as determined by SEM.

Movie S1: The real-time high resolution movie of the first lithiation process of MSC composite. 